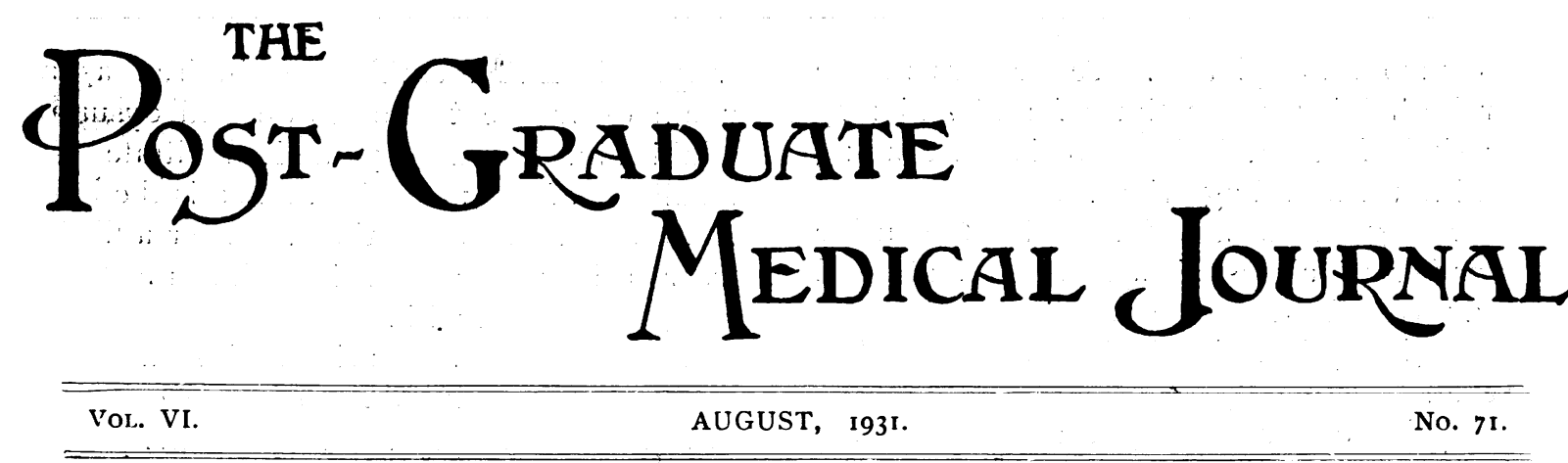

\title{
CONTENTS
}

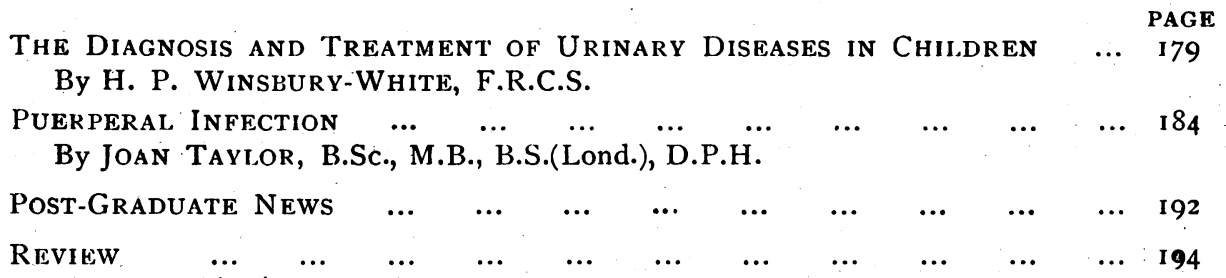

Fellowship of Medicine and Post-Graduate Medical Association.-

$\begin{array}{lllllllllll}\text { SPECIAL COURSES } & \ldots & \ldots & \ldots & \ldots & \ldots & \ldots & \ldots & \ldots & \ldots & 194\end{array}$

\section{THE DIAGNOSIS AND TREATMENT OF URINARY DISEASES IN CHILDREN.} By H. P. WINSBURY.WHITE,

\section{F.R.C.S.}

Senior Assistant Surgeon, East London Hospital for Children, and St. Paul's Hospital for GenitoUrinary Diseases; Urologist, St. John's Hospital, Lewisham.

DistURBanCES of micturition offer the chief cause for investigation of the urinary tract in children. Less commonly pain or swelling in one or both loins, or a pyrexia, having its origin in a renal infection, which has caused no striking urinary or local symptoms, are the reason for the enquiry. It is of some interest therefore to consider,urinary diseases in children from the point of view of these outstanding fealures. The cause of the last condition is generally at once apparent when the urine is examined.

Increased Frequency and Dysuria.-These two symptoms often óccur together and in attacks, and commonly have their origin in a mild infection originating as a balanitis or a urethritis in the male, or a vulvc-vaginitis or urethritis in the female. On cystoscopy some degree of inflammation is found localized to the trigone. In a male child with a marked phimosis the latter cause should be suspected and dealt with at once. On the other hand children already circumcised are also subject to ascending cystitis, but in these the infection originates in the urethra. It is only the exceptional case in which the urethritis is gonococcal in origin. As a consequence of congenital narrowing of the external urinary meatus in the male often there is to be seen a small crust on the exit. On removing this the injected urethral mucosa is at once apparent. The remedy is equally simple in this group, for a meatotomy followed by several urethral dilatations remedies the urethritis and cures the attacks.

When the latter are severe a little terminal hæmaturia, with the dysuria reaching its maximum as the last few drops of blood-stained urine are expressed, is not 
an uncommon picture. Tenderness in one or both loins is sometimes found and may mask the fact that the infection is of ascending and not descending origin.

If organisms are found in the urine Bacillus coli almost always predominate, and may wrongly strengthen the conclusion that the primary infection in the urinary tract is renal. Frequently the evidence in the urine of infection is so slight as to give the impression that this is not the cause of the symptoms, and only by cystoscopy is a mild inflammatory condition established as the cause. In female children vulvovaginitis must be sought most carefully, sometimes the discharge is very slight, clear, or only faintly turbid, exceptionally it is definitely purulent. Whatever the origin of the discharge in a particular case, unless the latter is definitely purulent, the gonococcus is not likely to be found. When purulent, smears and cultures should be made from the urethra and cervix. The latter structure can be viewed and approached, if a special tube is fitted on to an anterior urethroscope. This applies to infants, and no anæsthetic is required.

The way in which the lesser degrees of genital infection arise is often obscure. It frequently happens that while the discharge from the cervix is free from turbidity, although organisms such as $B$. coli and diphtheroid bacilli can sometimes be grown from it, yet some mucu-purulence is noted at that same time in the discharge lying in the vagina. It has been suggested, not without some appearance of justification, that a vaginitis arises as a deficiency disease. Small foreign bodies found in the vagina are sornetimes the cause.

The minor degrees of the complaint clear up readily with daily vaginal douching with boric lotion continued for a week, combined with attention to general dietetic and medicinal requirements, and it is generally gratifying to note the way the bladder symptoms disappear also.

Pyelonephritis of descending origin is frequently first identified in children as a result of frequent micturition and dysuria. $\frac{3}{\mathbb{D}}$ Fluid diuretics and potassium citrate till $\stackrel{\varrho}{c}$

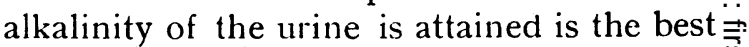
routine treatment for these. Later a likely extra urinary focus of infection must be $\frac{}{0}$ sought and dealt with.

The frequency, dysuria, and sometimes $\frac{\mathbb{\Omega}}{\Omega}$ terminal hæmaturia due to vesical calculus have the characters that they are persistent $\overrightarrow{0}$

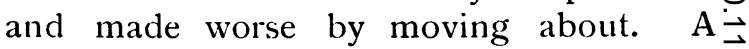
radiogram may overlook a small stone in the bladder, and cystoscopy should therefore be음 carried out, when the X-ray is negative and $\underline{3}$. stone is suspected. The majority of vesical ${ }^{\circ}$ calculi in children can with safety be treated ${ }_{-}^{-}$ by litholapaxy in expert hands. A large or very hard stone should be removed by supra-을 pubic lithotomy. A stone impacted in the posterior urethra may give rise to the abovementioned symptoms, but there is ultimately some urethral discharge, which becomes $\vec{\circ}$ blood-stained if the stone remains lowe enough. Such a calculus can often be pushed back into the bladder with a sound, when can be crushed. If this cannot be done it is better to do a suprapubic lithotomy and to\% remove the stone by combining urethral in $-\stackrel{\mathbb{Q}}{\circ}$ strumentation with dilatation of the internal $\overrightarrow{\overrightarrow{0}}$ urinary meatus, rather than to risk damage to the verum montanum by a perinealo section. The latter may be safely done, however, if the stone is impacted in the bulbus urethra.

Enuresis.-One discriminates several clinical types : those in which the symptoms have continued since birth; those in which theo symptoms have supervened after infancy is passed at some time during childhood.

In the latter group one can often put one's on finger on a definite predisposıng cause. Notr infrequently the condition comes on after one of the infectious diseases such as measles, scarlet fever, or diphtheria. In others a perito pheral source of mild chronic infection can be found, such as enteritis, bronchitis, tonsil: litis, atresia of the external urinary meatus, or vulvo-vaginitis. The mere fact of finding 
neither pus nor organisms in the urine when this happens to be examined does not shift the responsibility from one of such foci if it is present.

Then there is the more obvious association with infection when chronic pyuria is present. This is most commonly the result of congenital dilatation or lithiasis in some part of the urinary tract.

The mentally deficient and the nervous child are both types which are well known in association with enuresis. Some degree of spina bifida has been noted in other cases.

It is exceptional that a case does not fit into one of the above-mentioned groups. In a small proportion of cases the habit becomes established without any cause which can be revealed.

Before treatment for enuresis is undertaken, a careful history and a thorough general overhaul is essential, and any possible cause which is discovered should be dealt with. One would like to lay special emphasis on the minor, and often almost insignificant infective conditions about the genitals which may be of great importance, and yet may escape notice. The influence of a mild infection of the urethra and neck of the bladder, is admirably demonstrated by the frequent beneficial effect of the cystoscopy on the enuresis. For it generally ceases afterwards for a week or more. It is highly suggestive in such circumstances that the dilatation of the urethra and internal urinary sphincter, which takes place with the instrumentation, is an effective remedy for the infection. In certain cases this result indicates the necessity for a repetition of the dilatation at intervals determined by the effect of this procedure.

In cases which have not benefited from any form of treatment, the parents must make up their minds to control the bed wetting, first by discovering whether it occurs once or twice during the night, and at what times, and then to have the child lifted out an hour before this usually takes place. The taking of food and drink is kept as regular as possible with regard to the quantity and to the time of going to bed.

Tea, coffee, cocoa, and other diuretic drinks should be left out of the evening meal, and foods such as strawberries, rhubarb, asparagus, which are often irritating to the urinary passages, should be avoided altogether. Older children can often be taught to supervise themselves during the night by means of the alarm clock, until ultimately the habit disappears.

It is exceptional for the child not to gain complete voluntary control ultimately.

Hamaturia in children is one of the most interesting types of case for investigation.

The first point to determine is whether the blood is merely the expression of a few drops of blood-stained urine at the end of micturition, or whether the urine is equally discoloured throughout the act. If the former condition is established then it is from the neck of the bladder that the hæmorrhage arises, and the genital and lower urinary tracts should be examined carefully to ascertain whether one of the conditions $\mathbf{J}$ have already stressed is not present. One of these will generally be found. Less commonly it is the first striking manifestation of a pyelo-nephritis (pyelitis). It is only the very exceptional case when a vesical calculus is present. Cystoscopy should never be carried out while this symptom is acute. Fluids, diuretics, and sedatives are indicated until the attack has settled before any kind of instrumentation is undertaken.

If the hæmaturia is not terminal, time, and perhaps an unnecessary cystoscopy, will be saved, if the child's teeth and tonsils are first examined. In my experience hypertrophied and badly infected tonsils are the commonest cause in children of hæmaturia which does not occur at the end of micturition. Sometimes the blood has already been noted in the water for one or more weeks and generally clears within a week of tonsillectomy.

Removal of bad teeth, especially if these 
are associated with apical abscess is equally effective.

The presence of casts in the urine should strengthen rather than weaken the responsibility of the suspected septic focus.

In other cases of persisting hæmaturia in children, no such focus can be found. An antecedent infectious disease is often established, but this is not always the case, and the presence of casts justified the diagnosis of hæmorrhagic nephritis.

Hæmaturia from chronic renal infection, complicating hydronephrosis, or a more extensive dilatation of the upper urinary tract, is in my experience as common as the one just discussed, and renal calculus as a cause of hæmaturia is no less common. Radiograms, followed by intravenous pyelography when necessary, are essential to determine the presence of these conditions.

Hæmaturia of renal origin as a complication of acute pyelonephritis, is generally easy to recognize as the renal infection is accompanied by pyrexia, and initiated by some degree of rigor, which however may escape notice in a child. With a view to safeguarding against a recurrence, teeth, tonsils, respiratory and intestinal tracts in their turn must be suspected of supplying the infection to the kidney until the focus is found. The hæmaturia of renal tuberculosis is generally accompanied by persisting increased frequency and dysuria, but in exceptional cases the latter symptoms are insignificant, and recurring discoloration of the urine with blood calls for cystoscopy, and complete radiological and bacteriological investigation. The last method generally requires to be supplemented by guinea-pig inoculation.

In connection with the bacteriological examination a warning is not out of place that the urine is often secondarily infected with Bacillus coli, which may completely mask the presence of Tubercle Bacilli. Cystoscopy should, however, prevent this mistake.

In my experience these cases in children generally turn out to be bilateral, and therefore beyond surgical aid.

Polycystic kidney is sometimes the cause $\stackrel{\complement}{.}$ of hæmaturia. The presence of the condi- $\overrightarrow{\vec{F}}$ tion may be suspected if the characteristic $\frac{?}{0}$ lobulated renal tumours are palpated. The question can be settled by intravenous $\frac{\overline{\bar{s}}}{\bar{\sigma}}$ pyelography.

In renal sarcoma hæmaturia usuallyळ occurs so late in the disease that a palpable $\overrightarrow{0}$ renal swelling and wasting of the patient $\overrightarrow{\vec{\omega}}$ creates a typical clinical picture which at $\stackrel{\omega}{\sigma}$ once establishes the diagnosis.

Pyuria. - If this condition is known definitely to have persisted in any considerable degree for many weeks or longer, then it is certain that there is some gross patho- $\vec{v}$ logical condition present somewhere in the 음 urinary tract. Such a condition is incom- $\vec{D}$ patible with the appearance of good health, and these patients have a typically pale and $\stackrel{5}{\oplus}$ debilitated aspect.

Discrimination must be carefully mas between those cases in which it is definite known that the pus has persisted withou interruption over a prolonged period, and those in which pus is reported as being present both at a recent examination and at $\cong$ one carried out months or years before, $\stackrel{\overrightarrow{0}}{3}$ without any knowledge of the state of affairs $\frac{\partial}{\partial}$ in the intervening period. In the latter group the cases are frequently not chronic but recurring, and if so the aspect of the patient is that of good health, which is in contrast with the former group. The prognosis is good because a cure is generally not $₹$ difficult by dealing with some accessible 음 extra urinary focus of infection. In the $\frac{D}{O}$ chronic pyuria cases, a proper routine examination with the cystoscope, plain X-ray, and $\mathcal{O}$ an intravenous urogram, must be pursued 0 until the case is located.

In the upper urinary tract one or other of the following causes will be found : Stone,

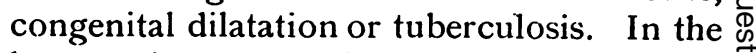
lower urinary tract it is usually some dis- $\vec{T}$ turbance of micturition rather than pyuria that dominates the clinical picture. 
Renal calculus almost without exception requires operation.' Children stand operations on the kidney extremely well. As a rule pyelo or nephro-lithotomy rather than nephrectomy meets the case. Congenital dilatation of the upper urinary tract provided it is unilateral generally required nephrectomy. My experience is that this operation is borne well even by children of 2 years. In renal tuberculosis, nephrectomy would only be considered when the disease can be shown to be unilateral. This is seldom the case in children.

Retention of Urine is uncommon in children but I have seen it in the following circumstances : As a complication of acute cystitis, as a result of a stone impacted in the urethra, as a complication of anterior poliomyelitis, as a part of a widespread dilatation involving the upper urinary tract and the bladder, quite independently of any obstruction in the urethra. As a result of obstruction to the internal urinary meatus from the presence of a sarcoma of the bladder. The condition has also been reported complicating phimosis, atresia of the external urinary meatus, urethral stricture and valves.

When there is no mechanical obstruction, catheterization accompanied by vesical irrigation is eventually necessary. A urethral stone must be removed by instrumental interference. An indwelling catheter, as a temporary measure when the bladder is badly infected, or a supra-pubic cystotomy may be required when the retention seems to be permanent.

Pain in Renal Region.-A complaint of pain in one or both renal regions may have its origin in varieties of causes, ranging from inflamnation of the overlying bowel. In the kidney itself, any of the pathological conditions already mentioned, may be responsible. The fact of the persistence of the symptom calls for a urological investigation until the explanation is clear.

In young children it is difficult and sometimes impossible to know whether the pain is central or lateral. This applies equally to spasms of colic, which may wrongly be thought to have an intestinal origin. Whatever the situation, or the nature of the pain, the upper urinary tract must always come up for careful consideration and a full sequence of diagnostic procedures pursued until the cause is established. The absence of any outstanding pathological elements in the urine should not deter the investigator from a full enquiry as to the condition of the urinary tract.

The ultimate onset of pain, frequently the result of an infective complication, often calls attention to a condition which is old standing. Colic is often renal but not due to stone, often being infective in origin or due to hydronephrosis. On the other hand small uric acid and ammonium urate calculi are not uncommon in children, and show such poor shadows on a radiogram that they may escape detection.

Palpable Enlargement of the Kidneys.-It is seldom that a renal tumour is the first feature to call attention to some gross kidney disease, but in certain cases of general ill-health an abdominal examination reveals renal enlargement on one or both sides. Hydronephrosis, polycystic kidney, renal sarcoma or perinephric abscess may at times be discovered in this way. But these together with most pathological conditions of the kidney, generally first attract attention by local pain or obvious changes in the urine, or disturbances of micturition.

Renal sarcoma is frequently inoperable when first discovered, and if not the prognosis is very bad as a result of nephrectomy. In polycystic kidney as the condition is generally bilateral, operation is inadvisable.

Perinephric abscess gives an obvious cystic mass, dull on percussion and associated with pyrexia. There may be no pathological elements in the urine. The condition responds readily to incision and drainage. 
Cystoscopy has been mentioned frequently in the course of this paper as a necessary diagnostic procedure. This will very properly raise the question as to whether it is a practicable procedure in infants and young children. One can say without hesitation that cystoscopes are made of such a fine calibre that they can be safely passed on infants, without fear of damage to the parts.

\section{PUERPERAL INFECTION.}

\author{
By JOAN TAYLOR, \\ B.SC., M.B., B.S.(LOND.), D.P.H. ${ }^{\prime}$
}

From the Obstetric Unit and Department of

Bacteriology, University College Hospital, London.

PUERPERAL infection includes all abnormal conditions resulting from the entrance of organisms into the genital tract during labour or the puerperium.

Puerperal infection has been recognized for many hundreds of years, and was mentioned by both Hippocrates and Galen in their works. Later, in the seventeenth century, Willis, in England, wrote a treatise on the subject as it occurred at that time. About the middle of the nineteenth century interest in this subject was aroused, as at that time about I $5_{5}$ per cent. of all patients delivered in institutions died of this disease. Semmelweis, working in Vienna, showed that puerperal infection was identical with wound infection. Pasteur cultivated organisms from cases, and later Lister showed the value of antiseptic methods in midwifery. From this short historical survey one realizes that it was not until the latter half of the nineteenth century that our present views on this subject began to emerge.

\section{BACTERIOLOGY.}

As early as the eighteenth century it was recognized that puerperal infection was con-

1 Working during part of the time with the aid of a grant from the Medical Research Council. tagious. It must be remembered that at this time the death-rate from sepsis was $\mathrm{I}$ in 6 in institutions owing to the rapid spread of epidemics, which are controlled to-day by the universal use of antiseptic methods. The contagious nature of the disease was not urged strongly until Oliver Wendell Holmes read his classical paper at Boston in 1843 . In this paper he showed that epidemics could always be traced to lack of cleanliness on the part of the attendants in charge of cases.

This work was not generally appreciated until as a result of the application of Lister's antiseptic methods there was a sudden fall in the number of cases infected. Much detailed bacteriology done during the whole of this present century goes to show that puerperal infection is due to the invasion of the genital tract by pyogenic organisms. As will be shown subsequently, any pyogenic organisms can give rise to infection. This is readily understood when we consider that the large raw area in the uterus, in continuity, through the vagina and open cervix, with the external surface, is comparable witho any raw area elsewhere in the body. Certain? organisms, such as the gonococcus, are more likely to infect the uterus than a surface wound, but taking all the known pyogenic organisms, as any one may infect a surface wound, so may any one, or more than one, infect the uterus.

Streptococci.-Streptococci may be classified as follows :-

Streptococci.-Streptococci may be classified as follows :Aerobic Anaerobic

hæmolytic non-hæmolytic

Schotmuller, in 1903, first differentiated between hæmolytic and non-hæmolytic streptococci, by showing that when grown on blood-agar plates, certain strains were surrounded by a clear zone of hæmolysis in which no red blood-cells were present, whereas other strains did not exhibit this property. The former are known as hæmolytic, and the latter as non-hæmolytic, streptococci. 